\title{
Consumer Perceptions in the Adoption of the Electronic Health Records in Australia: A Pilot Study
}

\section{Neethu Mathai}

School of Engineering and Information Technology

Murdoch University

Perth, Australia

Email: neethu.mathai@murdoch.edu.au

\section{Mohd Fairuz Shiratuddin}

School of Engineering and Information Technology

Murdoch University

Perth, Australia

Email: f.shiratuddin@murdoch.edu.au

\section{Ferdous Sohel}

School of Engineering and Information Technology

Murdoch University

Perth, Australia

Email: f.sohel@murdoch.edu.au

\section{Xuequn Wang}

School of Engineering and Information Technology

Murdoch University

Perth, Australia

Email: a.wang@murdoch.edu.au

\section{Abstract}

The paper reports an empirical investigation of the factors affecting consumer perceptions of the adoption of Electronic Health Records in Australia. This paper also details the processes involved in the pilot testing of the instrument where it has been pilot-tested to a convenience sample by sending individual postal survey envelopes to shortlisted community organisations in Australia.

Reliability analysis to check the internal consistency was performed using the Cronbach's alpha. Content validity was achieved by reviewing the instrument with a panel of experts. The results of this pilot study proved the feasibility of a full-scale study and these could be used as the basis for refinement of the instrument. Based upon the outcome of validity and reliability testing, items for the final instrument were identified. The findings showed that the tested model does fit the data well and has a significant and positive impact on the consumer's attitude in using the EHR.

Keywords consumer perceptions, electronic health record, my health record, pilot study, questionnaire validation 


\section{INTRODUCTION}

An Electronic Health Record (EHR) is defined as an electronic version of an individual's medical history and may include all of the key administrative, clinical data including, but not limited to information such as demographics; medications; past medical history; immunizations; laboratory data and radiology reports etc. (Rahman et al. 2015). Australia's EHR system was previously known as the Personally Controlled Electronic Health Record (PCEHR) or eHealth record, and now it is called the My Health Record. Personal electronic health records allow consumers to manage their own healthcare. As such, consumers would have control over the data and access functions of their own health records. Each record potentially contained a summary of a patient's health information and was accessible by both the consumer and any health professional approved for viewing (Torrens et al. 2017).

This paper presents a pilot study which is a work-in-progress of an upcoming full-scale study. In this paper, we present the methods and results of the pilot study; studying the factors affecting consumer perceptions on the adoption of the EHRs in Australia. In this pilot study, responses provided by 48 respondents were gathered. The results were used to improve the measures and conduct primary tests. Specifically, a survey was conducted to assess the feasibility of a full-scale study, and to evaluate the survey based on the participant's understanding and perception of the questionnaire items. Participants were requested to provide their feedback on the survey design, and any suggestions or comments they think would improve the survey. Furthermore, the survey helped us to evaluate the reliability of the instrument and examined the clarity and appropriateness of the questions.

\section{BACKGROUND}

The EHRs has the potential to address many of the challenges currently faced by healthcare systems. A well-designed EHRs could potentially reduce healthcare-related costs, improving its quality and efficiency and, most importantly prevent medical errors (Grana et al. 2015). Analysing whether the currently adopted EHRs improves quality and efficiency has important implications on how best to employ it. The EHRs, when optimally implemented, holds a tremendous potential benefit for the healthcare system, could enhance how patient data are documented, organized, and utilised (Bowman 2013).

A comprehensive analysis of the EHR consumers' perceptions and attitudes are key to a successful implementation. The move to personally controlled health records and changes to the healthcare system have reframed patients as 'consumers', with an assumption that they have a consumer's right to select and choose in the health marketplace and are participants in the development of their e-health record (Cripps et al. 2012). Consumers have an important role to play whereby they hold valuable, first-hand knowledge of what could contribute to the successful employment of an EHRs (McGinn et al. 2011). Examining consumer concerns particularly in the early stages of the EHRs implementation helps in gaining a deeper understanding of consumer views and provide important insights for its development (Andrews et al. 2014).

It is therefore important to find out what are the factors affecting consumer adoption of the EHRs to improve the understanding of how consumers perceive this technology. The main objective of this pilot test is to access the feasibility and to refine the measures which will be used in the full-scale study exploring the consumer perceptions in the adoption of EHRs in Australia.

\section{METHODS}

The research uses a theoretical framework based on Technology Acceptance Model (TAM) (Davis 1989). The application of TAM in health care context was examined by the study (Holden et al. 2010), and results indicate TAM predicts a substantial portion of the use or acceptance of health IT.

The study includes the core constructs in TAM: namely, Perceived Usefulness, Perceived Ease of Use, Subjective Norms and Attitude. Additional external variables were also adopted- namely, Perceived Health Literacy, Perceived Computer Anxiety, Perceived Self-efficacy and Perceived Barriers. In our study setting, Perceived Usefulness is conceptualised as the extent to which an individual believes that the use of EHRs could enhance their performance and Perceived Ease of Use denotes the degree to which an individual believes that the use of the EHRs will be effortless. Subjective Norms refers to the degree to which an individual perceives that others who are important to them believe they should use EHRs. (Davis 1989; Kim et al. 2017). Perceived Health Literacy can be defined as the degree to which individuals have the capacity to obtain, understand, use and communicate basic health information and services to make appropriate health decisions (Services 2000). Perceived Computer Anxiety expresses 
an individual's apprehension or fear when faced with the possibility of using a computer. Perceived Selfefficacy represents the self-evaluation by an individual of their capacity to use EHRs (Compeau et al. 1995; Jian et al. 2012). Demographic factors indicate the socioeconomic characteristics of the individual such as age, gender, education level, income and living area. Perceived Barriers refers to the barrier variables involved in the EHR adoption such as security, privacy and availability of resources.

A survey questionnaire was developed after a thorough review of the literature related to the EHRs. The survey was divided into eight sections. All these sections have a number of questions to evaluate the consumer's perceptions and attitude towards EHRs. The majority of the questions were closed Likertscaled and categorical questions, although blank spaces for writing comments and suggestions were included at the end of each section. Table 1 shows the sources where the questionnaire items were taken from. Changes to some wording were made so that they are appropriate to fit the EHRs context.

\begin{tabular}{|c|c|c|c|}
\hline Construct & $\begin{array}{l}\text { Questionnaire } \\
\text { Sources }\end{array}$ & Construct & $\begin{array}{l}\text { Questionnaire } \\
\text { Sources }\end{array}$ \\
\hline Perceived Usefulness & (Davis 1989) & Perceived Computer Anxiety & (Heinssen et al. 1987) \\
\hline Perceived Ease of Use & (Davis 1989) & Perceived Self-efficacy & (Compeau et al. 1995) \\
\hline Attitude & (Davis 1989) & Perceived Health Literacy & (Noblin et al. 2012) \\
\hline Subjective Norms & (Davis 1989) & Perceived Barriers & $\begin{array}{l}\text { (Miller 2016) (Perera et } \\
\text { al. 2011) (Showell 2017) }\end{array}$ \\
\hline
\end{tabular}

Table 1: Sources of the items in the questionnaire

The questionnaire was reviewed by experts to assess its validity and acceptability, to ensure that the questions were clearly articulated and truly reflected the construct that is intended to be measured. This has not only helped in cross-checking the substantive aspects of the survey, but also improved the overall style of the instrument as well. The final version of the questionnaire was then piloted to assess its reliability. This pilot survey was granted ethics approval from the university's Human Research Ethics Committee. Prospective participants in the pilot survey were restricted to Australian residents aged 18 and above, who have some level of awareness of the EHR system. The survey was entirely voluntary and anonymous.

A list of contacts of community organisations was gathered from the Australian community directory, considering the size and diversity of the organisations and anticipated EHR awareness of people in those locations. Eight shortlisted community organisations in Western Australia, Queensland, New South Wales and Victoria were then contacted by phone for their consent, and once consented, 150 hard copies of the pilot survey materials were then mailed out to them. The pilot survey was opened for two months, and during the survey period, two reminders were made to ensure an acceptable level of response rate.

\section{STATISTICAL ANALYSIS RESULTS}

This section presents the analysis of the results of this pilot study.

\subsection{Descriptive statistics results}

According to (Nulty 2008), the expected response rate for postal surveys is $33.3 \%$. In this pilot survey, 150 surveys were distributed, 48 questionnaires were returned, and 47 questionnaires were treated as "complete" as one of the surveys has missing data in one of the question. Therefore, the effective response rate can be calculated as:

Effective response rate $=$ Total number of complete questionnaires returned $/$ Total number of questionnaires distributed $=47 / 150 \times 100 \%=31.33 \%$.

Data were analysed using SPSS, and descriptive statistics (frequencies) of the data was calculated to describe the basic features of the data in this study. Table 2 shows the characteristics of the respondents of this pilot survey. 


\begin{tabular}{ll|ll}
\hline Characteristics & N & Characteristics & N \\
\hline Gender & & Gross household income level & \\
Male & 21 & Below $\$ 18,200$ & 3 \\
Female & 27 & $\$ 18,201-\$ 37$, ooo & 3 \\
Age & & $\$ 37,001-\$ 87,000$ & 17 \\
$18-35$ years old & 16 & $\$ 87,001-\$ 180,000$ & 12 \\
$36-65$ years' old & 31 & $\$ 180$, oo and over & - \\
65 years old and above & 1 & Prefer not to say & 13 \\
Prefer not to say & - & Living area & - \\
Education level & & Rural Area & 24 \\
Secondary (High) school & 1 & Regional town/city & 24 \\
TAFE education & 6 & Major city & - \\
University undergraduate & 21 & Other (please specify) & - \\
University postgraduate & 18 & Prefer not to say & \\
Prefer not to say & 2 & & \\
\hline
\end{tabular}

Table 2: Characteristics of the respondents

Table 3 shows the distribution of the respondents by region/state.

\begin{tabular}{lccc}
\hline Western Australia & Queensland & New South Wales & Victoria \\
\hline 24 & 9 & 10 & 5 \\
\hline
\end{tabular}

Table 3: Distribution by region/state

\subsection{Internal consistency analysis}

Reliability analysis using Cronbach's alpha was conducted to ensure that the collected data was reliable. An accepted rule of thumb is that an $\alpha$ value of 0.6-0.7 indicates acceptable reliability, and 0.8 or higher indicates good reliability. Cronbach's alpha is sensitive to the number of items in the scale and is also affected by sample sizes (Cronbach 1951; Voss et al. 2000). The 44-item questionnaire was acceptable to the participants and showed good internal consistency, i.e. with Cronbach's alpha values between o.62-0.89. The results indicated that seven out of eight measured variables had Cronbach's alpha indicators that exceeded the test value of 0.70 . Table 4 presents a summary of the results of the Cronbach's alpha coefficients of each variable.

\begin{tabular}{lll|llc}
\hline Variable & $\begin{array}{l}\text { Cronbach's } \\
\text { Alpha }\end{array}$ & $\mathbf{N}$ & Variable & $\begin{array}{l}\text { Cronbach's } \\
\text { Alpha }\end{array}$ & $\mathbf{N}$ \\
\hline Perceived Usefulness & 0.857 & 3 & Perceived Computer Anxiety & 0.754 & 2 \\
Perceived Ease of Use & 0.884 & 2 & Perceived Self-efficacy & 0.618 & 2 \\
$\begin{array}{l}\text { Perceived Health } \\
\text { Literacy }\end{array}$ & 0.724 & 4 & Perceived Barriers & 0.743 & 8 \\
Subjective Norms & 0.895 & 3 & Attitude & 0.862 & 2 \\
\hline
\end{tabular}

Table 4: A summary of the results of the Cronbach's alpha coefficients of each variable

\subsection{Regression analysis}

Simple linear regression is used to summarize and study the relationships between the variables. Results of the linear regression analysis suggest that Perceived Usefulness $(\beta=0.70, p<0.05)$, Perceived Ease of Use $(\beta=0.53, p<0.05)$, Subjective Norms $(\beta=0.47, p<0.05)$, Perceived Self-efficacy $(\beta=0.52$, $\mathrm{p}<0.05)$, and Resource Availability $(\beta=0.32, \mathrm{p}<0.05)$ exhibits a statistically significant relationship to the dependent variable Attitude.

Pearson correlation was used to measure the strength of linear association. Results of the Pearson's correlation shows that Perceived Usefulness ( $\mathrm{rp}=0.585, \mathrm{p}<0.01$ ), Perceived Ease of Use ( $\mathrm{rp}=0.516$, $\mathrm{p}<0.01$ ), Subjective Norms ( $\mathrm{rp}=0.472, \mathrm{p}<0.01)$, Perceived Self-Efficacy $(\mathrm{rp}=0.421, \mathrm{p}<0.01)$, and Resource availability ( $\mathrm{rp}=0.462, \mathrm{p}<0.01$ ) have a statistically significant correlation with dependent 
variable Attitude while Perceived Health Literacy, Perceived Computer Anxiety, Security and Privacy concerns exhibited no significant correlation with consumers' Attitude in adoption of the EHRs.

\subsection{Participants comments and expectations about EHRs}

In this pilot survey, the final section asked for participant's expectations, suggestions or comments about electronic health records adoption. The respondents mainly shared their suggestions and comments on the EHR's usefulness, security and privacy, the need for awareness, and their expectations about EHRs.

Majority of the respondents were positive about the usefulness of health record system and believed it would improve the quality of care; as stated by Participant 29, "EHR system, would be beneficial to everybody and would definitely help to keep my medical information up-to-date". Participant 2 added, "I think the EHR system is a great idea and would happily use it, provided there are strict and high standards of security for my health information". Participant 30 believed and stated, "Easy to use in the age of computers".

Participants expressed strong security and privacy concerns regarding unauthorised access, security and data breaches of the system. For example, participant 35 stated, "EHR system is a wise idea, but one's personal record should not be leaked". Participant 25 supported this point by stating, "I believe it is a good idea to keep all health records of all citizens but my biggest concern is the security of the personal data". Participant 36 stated, "Recent hacking of internet databases indicate data is not secure. Otherwise, the system will be beneficial".

Participants also expressed their concerns regarding the usage of data for secondary purposes. For example, participant 45 stated, ". For me data protection is crucial. Privacy is paramount. There is cancer in my family. Could that stop me from getting insurance? What about a nosy employee?..”. However, participants trust the government, but doubt the ability to securely protect the information, as stated by participant 7, "Even though I trust the government, I doubt that the frauds may find a way to access the data and use for improper usage".

Participants further indicated EHRs awareness was not sufficient as stated by Participant 44, "a general awareness of the EHR system is important to the public". Participant 47 added by stating, "more educational awareness; extra training info for seniors". Participant 22 added, "User awareness training should be conducted example through TV advertisement". Participant 46 recommended, "online educational video on how to use EHRs for consumers". Participant 22 added, "Before adopting the EHR system make an awareness of the benefits of using this to the public by conducting community seminar or others".

\subsection{Feedback about the questionnaire items, data quality and non-response}

In the pilot survey after each section, participants were asked to provide their comments on clarity and understandability of the questionnaire items. They were also asked whether any question they think will be helpful to include in the main survey. Participants responded to most of the survey questions as clear and understandable. One of the improvements suggested in the questionnaire by the respondents was to use the term "Electronic Health Record" instead of just using the 'EHR' abbreviation in the questionnaire to avoid confusions and for better understanding. Another valuable comment raised was to use simple terms in the instructions by removing vague words such as "perceptions" and "perceived" and to make the terms 'health resources' clearer to 'websites/articles/information' in Section B (Perceived Health Literacy). Some participants expressed their concerns about security and privacy and suggested to include more questions on the respective sections. Based on the overall feedback of the participants, relevant changes have made in the questionnaire and a few more questions related to the security and privacy of EHRs have added to the final instrument.

The 'Response Quality' refers to the number of questions answered, item omissions and quality of responses for open-ended questions. In this pilot survey, the average number of questions, respondents left unanswered was very small, i.e., only one missing data. When the average number of questions respondents did not answer is small, this is an indicator of a good quality survey (Couper \& Triplett, 1999). This pilot survey also received good quality of responses for open-ended suggestions/comments section. Longer responses to open-ended section would indicate detailed responses, which contributes to the quality of a survey method (Schaefer et al. 1998). Therefore, the 'Response Quality' of this pilot survey can be rated as good as the respondents have given good quality suggestions and detailed comments.

The tendency to non-response was assumed to be affected by the method of conducting this pilot survey via postal mail, and the demographics of the respondents such as age and income level. Based on this 
pilot survey results, categories such as older people above 65 years, people from the rural area, and rich people with an income level of $\$ 180,001$ and above, have less participation in this survey. It is worth pointing out that every study has its confounding variables and limitations, and the confounding effect cannot be completely avoided (Šimundić 2013).

\subsection{Discussion and conclusion}

The effectiveness of the EHR system is mainly dependent upon the consumers' acceptance. Acceptance can ultimately contribute to the active and continued use of the EHR system (Hanna et al. 2017). It is likely that the consumers will not adopt or will abandon the EHR if the system does not align closely with their attitudes, and expectations (Greenhalgh et al. 2010). It is therefore important to find out the factors affecting the consumers' attitudes towards the adoption of the EHR.

Conducting and gathering data for this pilot survey was a relatively expensive exercise, but it yielded important information about the feasibility of the main study and the reliability of the instrument. The internal reliability for the direct subscales yielded good values. Findings from this pilot study demonstrate the influence of factors Perceived Usefulness, Perceived Ease of Use, Subjective Norms, Perceived Self-efficacy, and Resource Availability on consumers' Attitude towards the adoption of the EHRs in Australia. These variables exhibit a positive and significant correlation with the dependent variable Attitude. Since the linear regression analysis results show a large $\mathrm{R}^{2}$ value $\left(\mathrm{R}^{2}=63.2 \%\right)$, it can be concluded that the tested model does fit the data well and confirms that it is feasible and appropriate to conduct the main study on a larger population. Further, the invaluable feedback received in the comments section confirms the importance of the future study.

One of the methodological limitations highlighted by this pilot survey is the difficulty in getting responses from paper-based surveys conducted via postal mail. Postal surveys tend to be resourceintensive in terms of labour and financial investment. Another limitation is in determining internal consistency and correlation given the small sample size. Larger sample size should hypothetically lead to more accurate or representative results. This strengthens the argument of the importance of conducting further reliability in the upcoming main study to determine and confirm the overall psychometric properties of this instrument.

As a significant proportion of the population currently uses electronic technologies, the emerging data collection approach based on the internet is a relatively cost-effective survey alternative which can collect large amounts of data from participants in a short time frame. Similarly, they also seem to be feasible and effective in collecting data on sensitive issues or access to individuals which are hard to reach or in distant locations (Regmi et al. 2016). Further, feedback was also received from the community organisations that helped in recruitment that participants said that would have preferred to respond via an online survey and hence the proposed new study will collect data via an online questionnaire. The target population for this cross-sectional survey will be Australian residents aged 18 and over.

A better understanding of consumer characteristics, attitudes, and beliefs will contribute to the research field and provide valuable insights into the factors that influence the acceptance of, or resistance to the technology. Further, understanding these factors will provide the ability for health educators to design meaningful materials for promoting the adoption of EHRs.

\section{REFERENCES}

Andrews, L., Gajanayake, R., and Sahama, T. 2014. "The Australian general public's perceptions of having a personally controlled electronic health record (PCEHR)," International Journal of Medical Informatics (83:12), pp 889-900.

Bowman, S. 2013. "Impact of electronic health record systems on information integrity: quality and safety implications," Perspectives in Health Information Management (10:Fall).

Compeau, D. R., and Higgins, C. A. 1995. "Computer self-efficacy: Development of a measure and initial test," MIS quarterly), pp 189-211.

Cripps, H., and Standing, C. 2012. "Building patient trust in electronic health records,").

Cronbach, L. J. 1951. "Coefficient alpha and the internal structure of tests," psychometrika (16:3), pp 297-334.

Davis, F. D. 1989. "Perceived usefulness, perceived ease of use, and user acceptance of information technology," MIS quarterly), pp 319-340. 
Grana, M., and Jackwoski, K. 2015. "Electronic health record: A review," IEEE), pp 1375-1382.

Greenhalgh, T., Hinder, S., Stramer, K., Bratan, T., and Russell, J. 2010. "Adoption, non-adoption, and abandonment of a personal electronic health record: case study of HealthSpace," BMJ (341).

Hanna, L., Gill, S. D., Newstead, L., Hawkins, M., and Osborne, R. H. 2017. "Patient perspectives on a personally controlled electronic health record used in regional Australia: 'I can be like my own doctor'," Health Information Management Journal (46:1), pp 42-48.

Heinssen, R. K., Glass, C. R., and Knight, L. A. 1987. "Assessing computer anxiety: Development and validation of the Computer Anxiety Rating Scale," Computers in Human Behavior (3:1) 1987/01/01/, pp 49-59.

Holden, R. J., and Karsh, B.-T. 2010. "The technology acceptance model: its past and its future in health care," Journal of biomedical informatics (43:1), pp 159-172.

Jian, W.-S., Syed-Abdul, S., Sood, S. P., Lee, P., Hsu, M.-H., Ho, C.-H., Li, Y.-C., and Wen, H.-C. 2012. "Factors influencing consumer adoption of USB-based Personal Health Records in Taiwan," BMC Health Services Research (12:1), p 277.

Kim, K. K., Sankar, P., Wilson, M. D., and Haynes, S. C. 2017. "Factors affecting willingness to share electronic health data among California consumers," BMC Medical Ethics (18:1), p 25.

McGinn, C. A., Grenier, S., Duplantie, J., Shaw, N., Sicotte, C., Mathieu, L., Leduc, Y., Légaré, F., and Gagnon, M.-P. 2011. "Comparison of user groups' perspectives of barriers and facilitators to implementing electronic health records: a systematic review," BMC Medicine (9) 04/28, 02/09/received, 04/28/accepted, pp 46-46.

Miller, S. 2016. "Evaluation of the Participation Trials for the My Health Record," Final Report. November).

Noblin, A. M., Wan, T. T., and Fottler, M. 2012. "The impact of health literacy on a patient's decision to adopt a personal health record," Perspectives in Health Information Management/AHIMA, American Health Information Management Association (9:Fall).

Nulty, D. D. 2008. "The adequacy of response rates to online and paper surveys: what can be done?," Assessment \& evaluation in higher education (33:3), pp 301-314.

Perera, G., Holbrook, A., Thabane, L., Foster, G., and Willison, D. J. 2011. "Views on health information sharing and privacy from primary care practices using electronic medical records," International Journal of Medical Informatics (80:2) 2011/02/01/, pp 94-101.

Rahman, R., and Reddy, C. K. 2015. "Electronic Health Records: A Survey," Healthcare Data Analytics (36), p 21.

Regmi, P. R., Waithaka, E., Paudyal, A., Simkhada, P., and Van Teijlingen, E. 2016. "Guide to the design and application of online questionnaire surveys," Nepal journal of epidemiology (6:4), p 640.

Schaefer, D. R., and Dillman, D. A. 1998. "Development of a standard e-mail methodology: Results of an experiment," Public opinion quarterly), pp 378-397.

Services, H. 2000. Healthy People 2010: Objectives for improving health (Part B: Focus areas 15-28). Appendices, (US Department of Health and Human Services.

Showell, C. 2017. "Barriers to the use of personal health records by patients: a structured review," PeerJ (5), p e3268.

Šimundić, A.-M. 2013. "Bias in research," Biochemia Medica (23:1) 02/15, 12/10/received, 01/10/accepted, pp 12-15.

Torrens, E., and Walker, S. M. 2017. "Demographic characteristics of Australian health consumers who were early registrants for opt-in personally controlled electronic health records," Health Information Management Journal (46:3), pp 127-133.

Voss, K. E., Stem, D. E., and Fotopoulos, S. 2000. "A comment on the relationship between coefficient alpha and scale characteristics," Marketing Letters (11:2), pp 177-191. 


\section{Copyright}

Copyright: (C) 2018 authors. This is an open-access article distributed under the terms of the Creative Commons Attribution-Non-commercial 3.0 Australia License, which permits non-commercial use, distribution, and reproduction in any medium, provided the original author and ACIS are credited. 